Conclusions Majority of procedures can safely be carried out as day case with associated reduced lengths of stay. The mean length of procedure under propofol was significantly shorter. Adding propofol procedures on to GA lists increased list capacity by approximately an additional 1 procedure per list. Both GA and propofol procedures are overall safe with low rates of complications and no procedure-related mortality. This safety data provides the platform to perform more complex endoscopy under propofol sedation, especially during the pandemic.

\section{HTH-5 COULD MACHINE LEARNING (ML) IMPROVE INDICES FOR PREDICTING OUTCOME OF AUGIB?}

Gaurav Nigam*, A Thakur, P Dhiman, K Oakland, J Grant-Casey, A Douds, V Jairath, D Clifton, M Murphy, A Stanley, S Travis, National GI bleed steering group. UK national GIB $M L$ group, UK

\subsection{6/gutjnl-2021-BSG.71}

Introduction Risk stratification scores for acute upper gastrointestinal bleeding (AUGIB) have limitations. ML models using multiple variables have the potential to improve predictive value.

Methods Patients undergoing at least one endoscopy were selected from the 2007 UK upper gastrointestinal bleeding (UGIB) audit ${ }^{1}$ for developing ML models predicting 28-day all-cause mortality and rebleeding. Input variables were divided into pre-endoscopy \& endoscopy features (demographics, presentation, co-morbidity, concomitant drugs, biochemical parameters, pre-endoscopic management and first endoscopy findings). Random forest with 100 estimators or trees was used as a prediction model. Shapely additive explanations $(\mathrm{SHAP})^{2}$ identified the most relevant features in each model. MissForest $^{3}$ imputed missing data. Outcome prediction from ML models for pre-endoscopy and combined pre-endoscopy
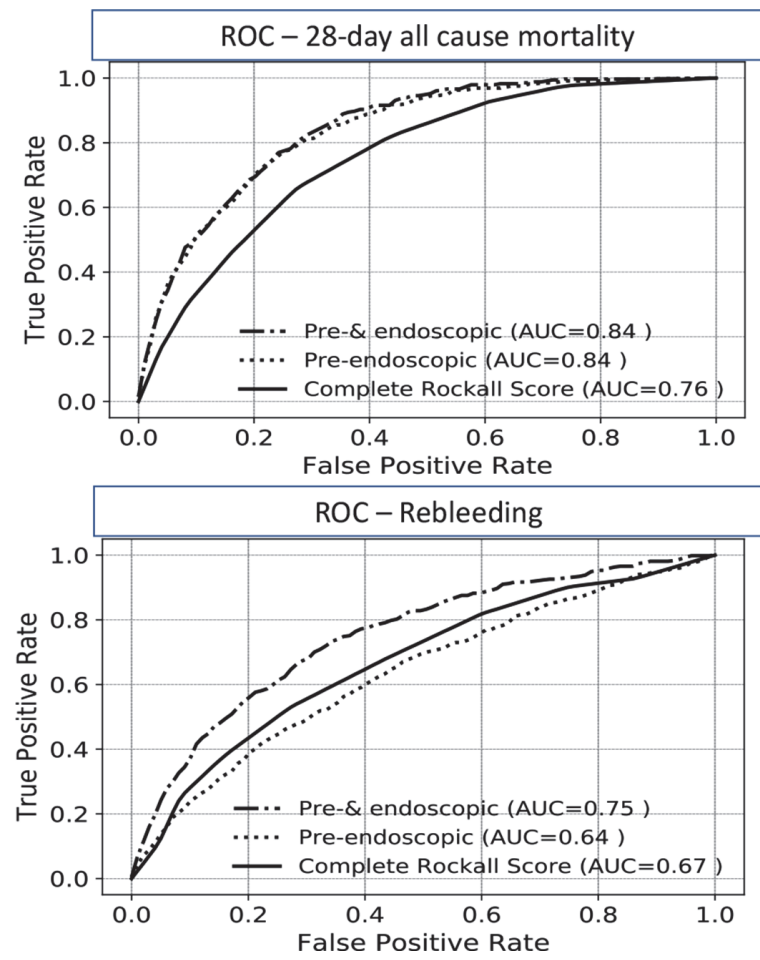

\& endoscopy were compared to Rockall scores. 5-fold crossvalidation compared performance, reported as average areas under receiver operating characteristic curve (AUC).

Results 5004 patients were included (mean age 66yr, 60\% male). 28-day all-cause mortality was $6.7 \%(n=335)$ with $9.3 \% \quad(n=465)$ rebleeding. All-cause mortality predicted by ML was independent of endoscopy (AUC 0.84, 95\% CI 0.820.85 , pre-endoscopy; 0.84 , 95\% CI $0.82-0.85$, pre- \& endoscopy) vs 0.76 , 95\% CI:0.75-0.77 for Rockall. Rebleeding predicted by ML was enhanced by endoscopy (AUC 0.64, 95\% CI: $0.63-0.66$ pre-endoscopy; AUC 0.75, 95\% CI:0.75-0.77 pre- \& endoscopy) vs AUC 0.67, 95\% CI:0.66-0.68 for Rockall. The SHAP tool reported 10 most relevant features (Figure 1).

Conclusion Pre-endoscopy ML models performed better than Rockall scores for predicting mortality. Endoscopy appeared to enhance ML prediction of rebleeding. Comparison with other risk scores was not possible from the dataset, but ML models from electronic records could develop dynamic decision support tools.

\section{REFERENCES}

1. Hearnshaw et al Gut 2011.

2. Lundberg et al Nat Mach Intell 2020.

3. Stekhoven et al Bioinformatics 2012.

\section{HTH-6 PREDICTORS OF LYMPH NODE INVOLVEMENT AND METASTASIS IN CRC CASES DIAGNOSED AT ST MARK'S HOSPITAL}

${ }^{1}$ Robert Kerrison*, 'Ms Sarah Marshall, ${ }^{2}$ Robert Kerrison. 'St Mark's Bowel Cancer Screening Centre, London, UK; ${ }^{2}$ University College London, London, UK

\subsection{6/gutjil-2021-BSG.72}

Introduction Colorectal cancer (CRC) is a leading cause of morbidity and mortality in England. Both 1- and 5-year
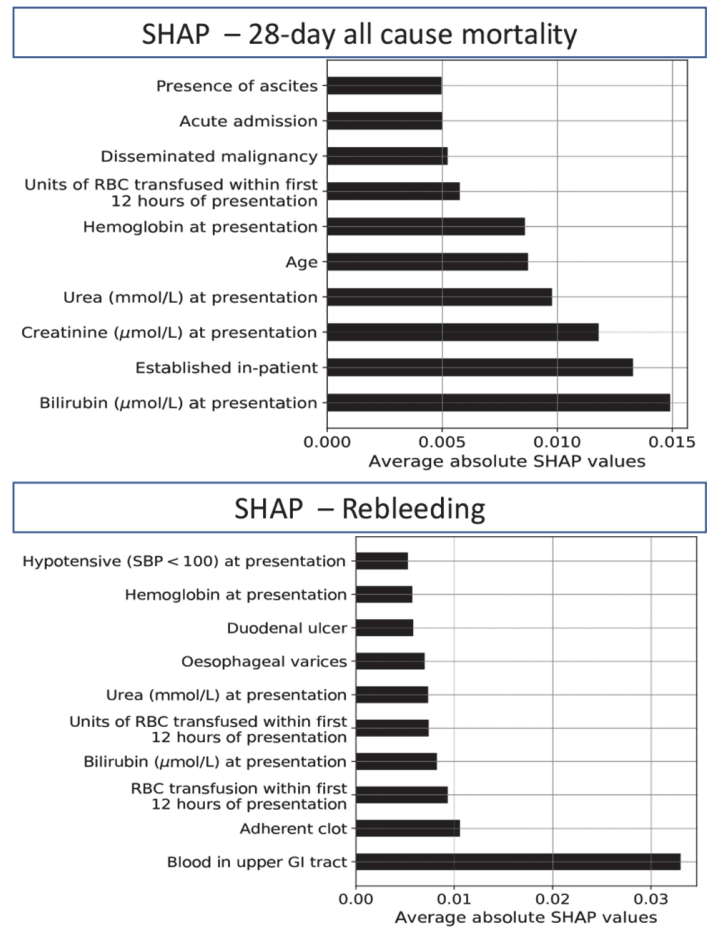
survival rates are greatly improved when the disease is diagnosed early; however, most cases are diagnosed late, and the prognosis for survival is generally poor. The aim of this study was to identify factors associated with receiving a late-stage diagnosis.

Methods We performed a clinical audit of 1,015 patients diagnosed with CRC at St Mark's Hospital between January 2017 and December 2020. Variations in Lymph node involvement and distant organs (at the time of diagnosis) were assessed using multivariate logistic regression. All analyses were performed using SPSS (Ver 27.0).

Results The sample was comprised of 584 (57.5\%) men and $431(42.5 \%)$ women, from a range of ethnic backgrounds: White British/Irish (413, 40.7\%), Other White background (67, 6.6\%), South Asian (176, 17.3\%), Other Asian background (84, 8.3\%), Any Black background (95, 9.4\%) and Any Other/Mixed background (42, 4.1\%). The mean age and Index of Multiple Deprivation score of patients were 70.29 years and 20.38, respectively. The most common route to diagnosis was GP referral (554, 54.6\%), followed by A\&E (218, 21.5\%), Other (150, 14.8\%) and Screening (93, 9.2\%). Data were missing for $279(27.5 \%)$ and 318 (31.3\%) individuals included in the predictors of lymph node involvement and predictors of distant metastasis analyses (respectively), meaning that $736(72.5 \%)$ and $697(68.7 \%)$ individuals were included.

The odds of lymph involvement decreased with age (aOR: 0.98 ; 95\%CIs: $0.97,1.00 ; \mathrm{p}=0.01$ ), were higher among South Asian adults compared with White British adults (aOR: 1.89; 95\%CIs: $1.09,3.28 ; \mathrm{p}=0.023$ ), and were lower among patients diagnosed through Screening or Other pathways compared with A\&E (aOR: 0.41, 95\%CIs: 0.24, 0.69; p=0.002 and aOR:0.40, 95\%CIs: $0.23,0.71 ; \mathrm{p}=0.015$, respectively). The odds of metastasis were higher among adults from Any Black Background compared with White British adults (aOR: 2.07, 95\%CI: 1.14, 3.88; $\mathrm{p}=0.018$ ), and were lower among those diagnosed through a GP referral or screening, when compared with those diagnosed through A\&E (aOR: 0.50, 95\%CIs: $0.32,0.79 ; \mathrm{p}=0.003$ and $\mathrm{aOR}: 0.14,95 \%$ CIs: 0.05 , $0.38 ; \mathrm{p}=0.001)$.

Conclusions Screening is associated with reduced odds of being diagnosed with CRC at a stage when lymph nodes and distant organs are affected, yet fewer cases are diagnosed via this pathway than any other. To determine whether there are missed opportunities to detect disease through screening, and whether inequalities in screening participation may account for the advanced stage at diagnosis observed for some ethnic minority groups, we will extract data on screening history.

\section{HTH-7 REDESIGNING IRRITABLE BOWEL SYNDROME (IBS) CARE IN NHS LOTHIAN (NHSL)}

${ }^{1}$ Leone Bissett ${ }^{*}$, ${ }^{1}$ Kerry Yuill, ${ }^{2}$ Alan Shand, ${ }^{3}$ Ken Trimble. ${ }^{1}$ Department of Dietetics, Leith Community Treatment Centre, Edinburgh; ${ }^{2}$ Department of Gastroenterology, Western General Hospital, Edinburgh; ${ }^{3}$ Department of Gastroenterology, Royal Infirmary of Edinburgh, Edinburgh

\subsection{6/gutjnl-2021-BSG.73}

Introduction IBS is one of the most common diagnoses made in gastroenterology clinics; however, for younger patients without alarm features, this diagnosis can be made in primary care. Service redesign to facilitate diagnosis and management

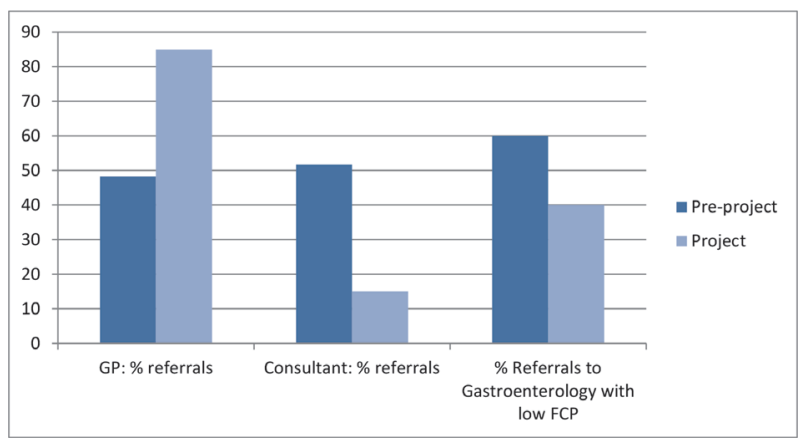

Abstract HTH-7 Figure 1

in primary care has the potential to release specialist capacity for more serious gastrointestinal (GI) disorders. A project was funded which aimed to promote a more confident diagnosis by general practitioners (GPs), reduce the number of referrals to gastroenterology services, provide timely dietetic intervention, optimise digital opportunities, promote supported patient self-management, and improve the patient experience.

Methods Gastroenterologists agreed an IBS diagnostic pathway incorporating ROME IV criteria, exclusion of alarm features, and appropriate investigations.

A Dietetic referral protocol was agreed by Dietitians, GPs and Consultant Gastroenterologists.

NHSL IBS steering group reviewed the IBS Post-Diagnostic Management and Referral Pathway.

Dietitians developed a video to provide 1st line dietary advice for patients with IBS.

A patient video feedback form was developed.

Consultant Gastroenterologists produced patient videos to provide information on IBS diagnosis and management.

A patient-initiated re-engagement form for further dietetic input following 1st line advice was included with the video.

Post-project surveys were circulated to key clinical staff involved in IBS management.

Results - Dietetic IBS referral source changed from GP 48.3\%/ Consultant - 51.2\% in 2017 to GP- 85\%/ Consultant - $15 \%$ during the project (Figure 1).

1. $19 \%$ reduction in the number of patients referred to GI with a low faecal calprotectin (FCP) (Figure 1).

2. The number of IBS referrals to dietetics increased.

3. 222 patients were sent 1 st line diet and IBS video link. 34 $(15.3 \%)$ re-engaged with the dietetic service.

4. Patient evaluation on the 1 st line video:

- $83 \%$ patients agreed/strongly-agreed that it was useful to have dietary information as an online video.

- $83 \%$ patients agreed/strongly-agreed that, after watching the video, they understood which dietary changes could manage their symptoms.

5. Gastroenterologist and GP feedback on the IBS Dietetic pathway was positive.

\section{Conclusions}

- An effective pathway for IBS diagnosis and dietetic treatment has been formulated.

- Increased opportunity for patient self-management.

- More timely IBS dietary advice.

- Practice has been standardised.

Reduced GI referrals for patients with low FCP. 